sequence. This conforms with the hypothesis of $\mathrm{C}$ fixation by $\operatorname{IgM}$ propounded by Ishizaka and Ishizaka (J. Immun., 102, 1337; 1969) and supported by electron micrographs of $19 \mathrm{~S}$ haemolytic antibodies forming loops to bind to nearby sites on a single erythrocyte membrane (Humphrey and Dourmashkin, Ciba Foundation Symposium on Complement, 1965).

Returning to the wider implications of their work, Humphries and McConnell, although aware of course that submembranous structures as well as lipid composition are involved in protein mobility in membranes, observe that the conditions of hapten mobility which they find necessary for C-mediated cytotoxic attack may also apply to direct lymphocyte stimulation by these liposomes. As evidence for this they point out that haptens stimulate an immunological response only when they are bound to a protein carrier. Such intriguing parallels will no doubt leave cellular immunologists asking how long it will be before the complementary experiments of manipulating the composition and hence the mobility or function of receptors is accomplished in a living cell.

\section{Circadian rhythms}

bv Miranda Robertson

The Dahlem Workshop on the

Molecular Basis of Circadian Rhythms was held in West Berlin on November 3-7.

WITH a field in the state that prevails in research on circadian rhythms, the particular format of the Dahlem Konferenzen should have proved an interesting experiment. The Workshops, of which this was the third, are explicitly designed to define the most important problems in a given field and to suggest fruitful directions for further research. Most of the conference time is spent in discussion: instead of being formally presented, background papers are written by and distributed to participants before the beginning of the meeting, and by the end, the members of each of six small groups have been forced into some kind of agreement in the production of a group report on their conclusions.

Agreement is a rare commodity in this field, whose present state has been compared (optimistically?) with that of Mendelian genetics before the discovery of the genetic code in the sense that the "hard" data are phenomenological. It is now very well established that numerous physiological processes, from photosynthesis in plants to motor activity in mammals, have an autonomous more-or-less 24-hour cycle which can be synchronised with the diurnal light-dark cycle by entrainment. The phenomenology has been enormously extended in experiments on how the various rhythms respond to changes in the phase or the period of the entraining cycle (the Zeitgeber). The task of the workshop participants was to decide on a strategy for discovering what cellular mechanism might give rise to oscillations with the observed characteristics. A group of mathematical modellers tried to decide what those characteristics say about how the clock works in principle and concluded that it is still impossible to distinguish between mechanisms as simple as the "dripping faucet" and as sophisticated as a limit cycle. Indeed, in general the field suffers from a surfeit of models and a dearth of critical experiments. Three categories of nonmathematical model were represented: enzyme models, genetic models and membrane models. The evidence in favour of any of them however tended to be somewhat general: substances which, say, disrupt protein synthesis or alter membrane characteristics sometimes disrupt the circadian rhythm one way or another.

One serious problem facing genetic and membrane modellers is that neither genetic regulation nor membrane function is properly understood in eukaryotic cells.

Another more general problem is that most researchers now agree that it is highly unlikely that there is any single clock mechanism. (Thus the analogy with Mendelian genetics is weak and Colin Pittendrigh's (Stanford) hopes of a "clock phage" doomed to disappointment.) Yet the models are built on the basis of data gleaned from the entire flora and fauna of biological horology, and the confusion is most apparent when anyone attempts to rule out any one possibility. For example, the importance of Ashkenazy's recent discovery is supposed to be that it eliminates genetic mechanisms, since circadian oscillations in enzyme activity seem to persist in red blood cells and

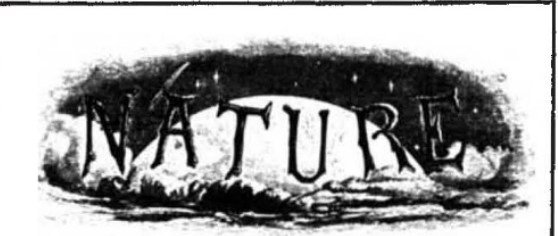

M. Charbonnier advocates .... the theory that people may subsist without food because the nitrogen from the air can be admitted into the circulation system, when the body has been emaciated by long abstinence. Feeding on air is an economical way of keeping soul and body together.

from Nature, 13, 95; Dec. 2, 1875. even their isolated membranes. But strictly, it rules out genetic mechanisms only for red blood cells.

This is a curiously tantalising field, in which promising leads seem to turn into blind alleys. The single-celled gonyaulax, for example, manifests circadian rhythmicity in three different physiological mechanisms, all of whose rhythms can be affected by a single mutation. Clock mutants are known in several other species, including two of Drosophila. Since both the genetics and the circadian phenomenology of Drosophila are so well known, one would expect such mutants to offer a golden opportunity for research.

It does seem, however, that the field would be best served if people stopped looking for "the" circadian clock and focused on that of one particular organism. It may not turn out to be a 'phage' but one well known mechanism could nonetheless prove in the end more illuminating than any number of plausible models.

\section{Sperm egg interaction}

\section{from a Correspondent}

The First Symposium of the International Society for Invertebrate Reproduction (ISIR) was held at Calicut on September 10-12, under the auspices of the University of Calicut and the Kerala Academy of Biology. Abstracts have been published in Invertebrate Reproduction, (edit by Adiyodi, K. G., and Adiyodi, R. G., Publication Division, ISRPI Secretariat, Calicut University, Kerala, India).

NINETEEN out of twenty animal species on our planet are invertebrates but it is probably no exaggeration that nineteen out of twenty papers published on reproduction deal with vertebrates. As knowledge of reproductive processes and mechanisms is essential for animal management and invertebrates as a group are at least as vital to human welfare as vertebrates, the Calicut Symposium therefore, marks a welcome departure.

Contributions to the symposium covered many aspects of sexuality and reproduction in invertebrates, such as sexual physiology and population dynamics, spermatogenesis, sperm egg interactions, ovarian cycles, chemistry of egg envelopes, nature of accessory sex glands and their secretions, reproductive behaviour and control mechanisms. The ground covered was so diverse that emphasis will be laid here only on contributions in sperm egg interaction.

Richard L. Miller (Temple Univer- 